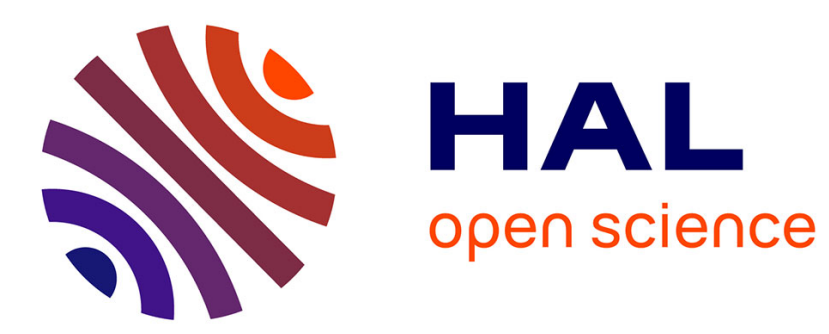

\title{
Renewal models for earthquake predictability
}

E. Garavaglia, E. Guagenti, R. Pavani, L. Petrini

\section{- To cite this version:}

E. Garavaglia, E. Guagenti, R. Pavani, L. Petrini. Renewal models for earthquake predictability. Journal of Seismology, 2009, 14 (1), pp.79-93. 10.1007/s10950-008-9147-6 . hal-00535488

\section{HAL Id: hal-00535488 \\ https://hal.science/hal-00535488}

Submitted on 11 Nov 2010

HAL is a multi-disciplinary open access archive for the deposit and dissemination of scientific research documents, whether they are published or not. The documents may come from teaching and research institutions in France or abroad, or from public or private research centers.
L'archive ouverte pluridisciplinaire HAL, est destinée au dépôt et à la diffusion de documents scientifiques de niveau recherche, publiés ou non, émanant des établissements d'enseignement et de recherche français ou étrangers, des laboratoires publics ou privés. 


\title{
Renewal models for earthquake predictability
}

\author{
E. Garavaglia • E. Guagenti • \\ R. Pavani · L. Petrini
}

Received: 11 December 2007 / Accepted: 23 December 2008 / Published online: 3 April 2009

(C) Springer Science + Business Media B.V. 2009

\begin{abstract}
The new Database of Italy's Seismogenic Sources (Basili et al. 2008) identifies areas with a degree of homogeneity in earthquake generation mechanism judged sufficiently high. Nevertheless, their seismic sequences show rather long and regular interoccurrence times mixed with irregularly distributed short interoccurrence times. Accordingly, the following question could naturally arise: do sequences consist of nearly periodic events perturbed by a kind of noise; are they Poissonian; or short interoccurrence times predominate like in a cluster model? The relative reliability of these hypotheses is at present
\end{abstract}

Electronic supplementary material The online version of this article (doi: 10.1007/s10950-008-9147-6)

contains supplementary material, which is available to authorized users.

E. Garavaglia $(\varangle) \cdot$ E. Guagenti $\cdot$ L. Petrini Department of Structural Engineering, Politecnico di Milano, Piazza L. da Vinci 32, 20133, Milan, Italy e-mail: garava@stru.polimi.it

E. Guagenti

e-mail: guagenti@stru.polimi.it

L. Petrini

e-mail: lorenza.petrini@polimi.it

R. Pavani

Department of Mathematics, Politecnico di Milano,

Piazza L. da Vinci 32, 20133, Milan, Italy

e-mail: raffaella.pavani@polimi.it a matter of discussion (Faenza et al., Geophys J Int 155:521-531, 2003; Corral, Proc Geoph 12: 89-100, 2005, Tectonophysics 424:177-193, 2006). In our regions, a statistical validation is not feasible because of the paucity of data. Moreover, the classical tests do not clearly suggest which one among different proposed models must be favoured. In this paper, we adopt a model of interoccurrence times able to interpret the three different hypotheses, ranging from exponential to Weibull distributions, in a scenario of increasing degree of predictability. In order to judge which one of these hypotheses is favoured, we adopt, instead of the classical tests, a more selective indicator measuring the error in respect to the chosen panorama of possible truths. The earthquake prediction is here simply defined and calculated through the conditional probability of occurrence depending on the elapsed time $t_{0}$ since the last earthquake. Short-term and medium-term predictions are performed for all the Italian seismic zones on the basis of datasets built in the context of the National Projects INGV-DPC 2004-2006, in the frame of which this research was developed. The mathematical model of interoccurrence times (mixture of exponential and Weibull distributions) is justified in its analytical structure. A dimensionless procedure is used in order to reduce the number of parameters and to make comparisons easier. Three different procedures are taken into consideration for the estimation of the 
parameter values; in most of the cases, they give comparable results. The degree of credibility of the proposed methods is evaluated. Their robustness as well as their sensitivity are discussed. The comparison of the probability of occurrence of a Maw $>5.3$ event in the next 5 and 30 years from January 1, 2003, conditional to the time elapsed since the last event, shows that the relative ranking of impending rupture in 5 years is roughly maintained in a 30-year perspective with higher probabilities and large fluctuations between sources belonging to the same macro region.

Keywords Renewal process - Hazard rate • Earthquake predictability $\cdot$ Credibility and robustness of the model $\cdot$ Italy

\section{Introduction}

One of the main issues in seismology and seismic engineering is reliable earthquake prediction. However, since earthquake occurrence depends on many random variables whose characteristics are often not well-defined, its prediction is still a difficult matter. Several approaches have been presented, but it seems that none is clearly superior.

The degree of uncertainty, epistemic and statistic, contained in the models leads to statements of this kind. "Although seismology has intensively analysed the characteristics of single earthquakes with great success, a clear description of the properties of seismicity ... is still lacking" (Corral 2006); “...test based on the likelihood ratios should be approached with caution..." and "physical significance (of cyclic effects and clustering) therefore remains questionable" (Vere-Jones and Ozaki 1982); “...these results... could be very relevant in the debate of seismic gap models versus clustering occurrence" (Corral 2005).

One of the major challenges is to interpret two apparently opposite characteristics in recurrence time models: temporal clustering and nearly periodic events. In Corral (2006), the author quotes "... results turn out to be in contradiction with each other, from the paradigm of regular cycles and characteristic earthquakes to the view of to- tally random occurrence" and proposes a unifying description of seismicity.

We do not have the pretension to enter in the debate, but we propose an elementary model that could be a first step to incorporate quasi-periodic events as well as Poissonian or clustered events. Moreover, we adopt an indicator, called credibility, to compare the competing models.

The mathematical tool of the model consists in a renewal process, already proposed by many authors in order to overcome the Poisson models. The renewal process is not free from criticism: (1) all past history, not only the last event, influences the earthquake generation; (2) the complexity of Italian context makes debatable a complete energy release after each earthquake followed by a new cycle; (3) the recurrence times are independent identically distributed (i.i.d.); (4) the sum of many causes of seismicity can suggest the Poisson process (this criticism being feeble because the renewal process includes the Poisson process as a particular case).

Nevertheless, in the frame of the renewal processes, the role of the specific survivor function (complementary distribution function) with the implied hazard rate is crucial. The mixture exponential-Weibull distribution function we propose has the capacity to incorporate in a probabilistic way three different behaviours (with different kinds of energy release): to collapse into a Poisson process, to model characteristic or clustering events.

The existence of characteristic or clustering events needs sound physical basis to be asserted; here the terms are used, for shortness, to indicate, respectively, the presence of rather regular temporal sequence (even if mixed with irregular short interoccurrence times) or the dominance of short interoccurrence times.

The main parameter responsible for making the mixture suitable in the three mentioned cases is the shape parameter $\alpha$ of the Weibull component. In general, its behaviour is as follows.

If $\alpha$ is greater than unity, the hazard rate comes out to be increasing until a peak value; it models a kind of seismic gap. If $\alpha$ is lesser than unity, the hazard rate comes out to be decreasing like in clustering events. If $\alpha$ is equal to unity, the proposed renewal process weakly differ from Poisson 
process. Moreover, the renewal process can collapse precisely in a Poisson process, as shown in the next sections.

In the SIS Intermediate Conference 2007 Risk and Prediction held in Venice in June 2007, such a model was already presented (Garavaglia et al. 2007) in a preliminary way; in this paper, it is studied and applied to the Italian seismic zones.

During the last 12 years and at present, Italian researchers are improving the earthquake catalogues and proposing different seismogenetic models of the seismic source. Seismic zoning, as well as the seismic catalogues, are again under revision.

In the context of a research plan proposed by Italian Department of Civil Protection (DPC) and National Institute of Geophysics and Vulcanology (INGV) (National Projects INGV-DPC 20042006) in the frame of which this work has been developed, new databases were released with the aim of calculating the probability of occurrence of strong events in the next 30 years. Earthquake sub-catalogues were obtained by the association of the events of the Italian historic catalogue CPTI04 (Catalogo Parametrico dei Terremoti Italiani-Versione 2004, INGV, Bologna; CPTI Working Group 2004) with seismogenetic source areas (SA; DISS03, INGV; DISS Working Group 2006) that are sources having homogeneous behaviour. For a lot of SAs, the number of events in the sub-catalogues is inadequate for a significant statistical analysis; therefore, the predictability could not be applied to the whole Italian context. In order to overcome this problem, the SAs have been grouped in macro regions (MR; Basili 2007) with the following characteristics: similar style of faulting and amount of deformation; this last association permitted to have adequate interoccurrence times datasets. The approach herein proposed was applied to these catalogues, as described in the following.

\section{The interoccurrences time model}

This paper is aimed at providing a model for earthquakes prediction, which takes into account interoccurrence time both for nearly periodic, characteristic earthquakes and for clusters and other randomly occurring earthquakes having a magnitude greater than a selected threshold.

The procedure is purely probabilistic, but at least three physical hypotheses are included:

1. a double behaviour in earthquake generation, as already said;

2. a dependence on the past history, expressed with the use of a renewal process;

3. a stationary asymptotic behaviour of the hazard: a very long seismic silence in a zone could be reasonably interpreted as an energy release having occurred somewhere else, rather than a continuous enormous accumulation of energy in progress in the zone; so even in the case of increasing hazard rate, its limiting value is a finite quantity.

More precisely, property 2 means that the probability of an earthquake occurrence depends on the elapsed time $t_{0}$ from the last event. Property 3 means that, in case of $\alpha>1$, the hazard increases with $t_{0}$ but, after a peak value, if the earthquake does not occur, it decreases and reaches a stable value, describing for every time increment a constant probability of an immediate occurrence.

In our regions, an exponential-Weibull (ex-w) mixture with the Weibull's shape parameter $\alpha>1$ appears to be suitable: it generally shows, after a first phase of weakly decreasing hazard rate, a central phase of increasing hazard followed by a stationary one. The exponential distribution mainly models the short interoccurrence times, related to clustering or random events, whilst the Weibull distribution mainly models the interoccurrence times related to the characteristic earthquake. Nevertheless, both distributions are defined in [0, $\infty$ ) without a threshold of separation between the two families of events. The weaker the characteristic earthquake is expressed, the more the tails of the two distributions overlap.

In any case, the mixture distribution overcomes the drawback of a single distribution that in general is not able to fit the entire class of interoccurrence times. In fact, what in general happens is that a distribution that fit well the short interoccurrence times does not fit the long interoccurrence time and vice versa. 
Moreover, note that the entire set of interoccurrence times has often coefficient of variation $(\mathrm{COV})$ near to 1 . So this property does not indicate a propensity towards characteristic earthquake nor towards clustering. The preliminary check of the global COV simply would indicate that the Poisson hypothesis is not rejectable. A better fitting is obtained through a mixture. The coefficient of aperiodicity (Matthews et al. 2002) of the resulting process can adequately fit sequences with different degrees of regularity.

The choice of the two component distributions here proposed for the mixture is the result of investigations on different mix of distributions and on correspondence between the theoretical characteristic of the mixture and the physical aspect of modelled phenomenon (Guagenti Grandori et al. 1990).

The ex-w survivor function, $\mathfrak{\Im}_{\tau}\left(t_{0}\right)=1-F_{\tau}\left(t_{0}\right)$, and the probability density function, $f_{\tau}\left(t_{0}\right)$, are, respectively (Cox 1962):

$$
1-F_{\tau}\left(t_{0}\right)=(1-p) \exp \left[-b t_{0}\right]+p \exp \left[-\left(\rho t_{0}\right)^{\alpha}\right],
$$

$$
\begin{aligned}
f_{\tau}\left(t_{0}\right)= & (1-p)\left\{b \exp \left[-b t_{0}\right]\right\} \\
& +p\left\{\alpha \rho\left(\rho t_{0}\right)^{\alpha-1} \exp \left[-\left(\rho t_{0}\right)^{\alpha}\right]\right\} .
\end{aligned}
$$

Let us call $\tau$ the interoccurrence time random variable, $t_{0}$ being its generic value, i.e. the time since the last event. The parameters involved in Eq. 1 are $p$, which can be considered the weight of the characteristic earthquake portion of the model; $\alpha$, whose value governs the Weibull coefficient of variation $\mathrm{COV}_{\mathrm{W}}$ :

$\operatorname{COV}_{\mathrm{w}}=\left\{\frac{\Gamma\left(1+\frac{2}{\alpha}\right)}{\left[\Gamma\left(1+\frac{1}{\alpha}\right)\right]^{2}}-1\right\}^{\frac{1}{2}} ;$

$b$ and $\rho$ that can be defined as functions of $\mu_{1}$ and $\mu_{2}$, which are the two return period components of the short interoccurrence times and of the characteristic earthquake interoccurrence times, respectively:

$\mu_{1}=\frac{1}{b} \quad \mu_{2}=\frac{\Gamma\left(1+\frac{1}{\alpha}\right)}{\rho}$.
Moreover, the COV of the mixture in the resulting process, called coefficient of aperiodicity, comes out to be:

$\operatorname{COV}_{\mathrm{ex}-\mathrm{w}}=\left\{(1-p) \cdot 2 k_{1}^{2}+p k_{2}^{2} \frac{\Gamma\left(1+\frac{2}{\alpha}\right)}{\Gamma^{2}\left(1+\frac{1}{\alpha}\right)}-1\right\}^{\frac{1}{2}}$.

The information about aperiodicity contained in Eq. 5 differs from the information contained in the sample COV.

The mixture (1) models a wide class of earthquake interoccurrence times; its hazard rate (HR), $\lambda\left(t_{0}\right)$, is defined as:

$\lambda\left(t_{0}\right)=\frac{f_{\tau}\left(t_{0}\right)}{1-F_{\tau}\left(t_{0}\right)}$

and it is shaped as required for property 3 . Because parameter $p$ can assume different values, the distribution (1) can explore different degrees of characteristic earthquake evidence: increasing values of $p$ means larger and larger evidence of characteristic earthquake; $p=1$ means lack of the irregular short interoccurrence times. In the two extreme cases $p=0$ and $p=1$, the mixture distribution (1) becomes, respectively, exponential and Weibull and property 3 fails: when $p=0$, the HR is constant in time and the predictability is missing; when $p=1$, the HR is continuously increasing until the certainty of an immediate occurring earthquake.

Equation 6 furnishes the prediction in a very short (infinitesimal) next time interval $\mathrm{d} t$ :

$$
\begin{aligned}
\lambda\left(t_{0}\right) & =\lim _{\Delta t_{0} \rightarrow 0+} \frac{\operatorname{Pr}\left\{t_{0}<\tau \leq t_{0}+\Delta t_{0} \mid \tau>t_{0}\right\}}{\Delta t_{0}} \\
& =\frac{f_{\tau}\left(t_{0}\right)}{1-F_{\tau}\left(t_{0}\right)}
\end{aligned}
$$

whilst the medium-term $\Delta t$ prediction is the probability, at each $t_{0}$ of an earthquake in the next $\Delta t$ :

$P_{\Delta t \mid t_{0}}=\frac{F_{\tau}\left(t_{0}+\Delta t\right)-F_{\tau}\left(t_{0}\right)}{1-F_{\tau}\left(t_{0}\right)}$.

In this context, prediction means, at each instant, the conditional probability of an earthquake with magnitude greater than a specific value in the next time interval $(\mathrm{d} t$ or $\Delta t$ ), given the length of the preceding seismic gap. 
The presence of four parameters is the main drawback of Eq. 1 in the estimation procedure. To avoid this shortcoming, we propose the following time scale transformation:

$h=\frac{t_{0}}{\mu}$

where:

$\mu=(1-p) \mu_{1}+p \mu_{2}$

is the global return period of the earthquake process. Equation 9 transforms Eq. 1 into dimensionless terms as follows:

$$
\begin{aligned}
1-F(h)= & (1-p) \exp \left[-\frac{h}{k_{1}}\right] \\
& +p \exp \left\{-\left[\Gamma\left(1+\frac{1}{\alpha}\right) \frac{h}{k_{2}}\right]^{\alpha}\right\}
\end{aligned}
$$

where $k_{1}=\mu_{1} / \mu, k_{2}=\mu_{2} / \mu$ are the two return period components, $\mu_{1}$ and $\mu_{2}$, expressed in dimensionless terms.

Equation 11 offers the advantage of presenting the range of a source's possible earthquake behaviour independently of the numerical value of the respective return periods. Then Eq. 10 becomes:

$1=(1-p) k_{1}+p k_{2}$

The parameter $p$ can now be estimated through Eq. 12 as follows:

$p=\frac{1-k_{1}}{k_{2}-k_{1}}$.

Three parameters remain to be estimated in Eq. 11. In order to make the estimate procedure easier and more stable, we proceed assuming $\alpha=$ constant with different trial values; then we choose the best one in respect to likelihood function. In this way, only $k_{1}$ and $k_{2}$ have to be estimated: they offer the advantage of being directly suggested by the sample, at least if the ratio $r=$ $k_{2} / k_{1}$ is large enough to ensure weak overlap of the two component densities. In this case, the mean values of the interoccurrence times $<1$ and the interoccurrence times $>1$ give empirical estimates of $k_{1}$ and $k_{2}$, respectively.

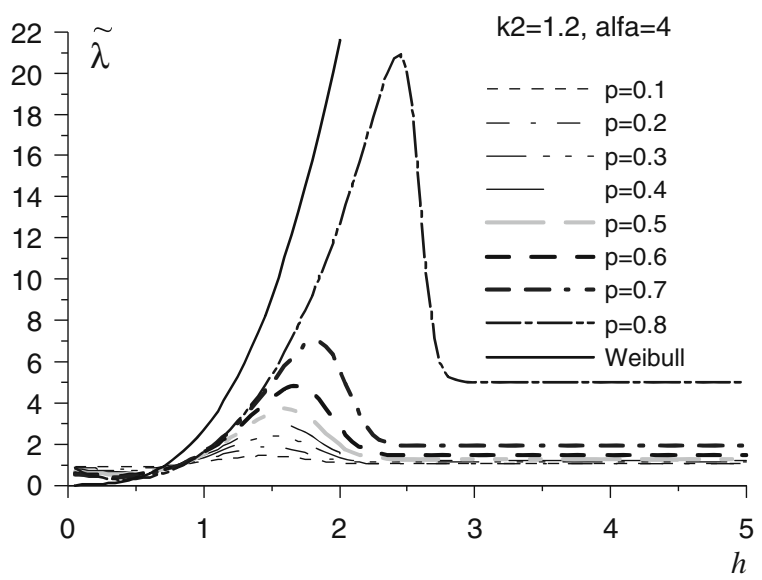

Fig. 1 Dimensionless HR for conjectural "truths" with $k_{2}=1.2$ and $\alpha=4$

Varying $k_{2}$ and $k_{1}$, we obtain different families of earthquake processes, representing conjectural "truths". In Figs. 1, 2, 3, 4 and 5, they are shown in a compact form through the dimensionless HR $\tilde{\lambda}$.

The assumed range [1.2-2] for $k_{2}$ can be considered exhaustive for a wide class of Italian seismic zones. The values of $k_{1}$ are chosen between 0 and 1 to give, from Eq. 13, equally spaced $p$ values $\left(0.1 \leq p<1 / k_{2}\right.$ with increment 0.1$)$.

In the figures, the value of $\alpha$ is 4 . Our large set of numerical simulations show that larger or smaller values of $\alpha$ lead to similar behaviours with more or less pronounced peak relative to characteristic earthquake.

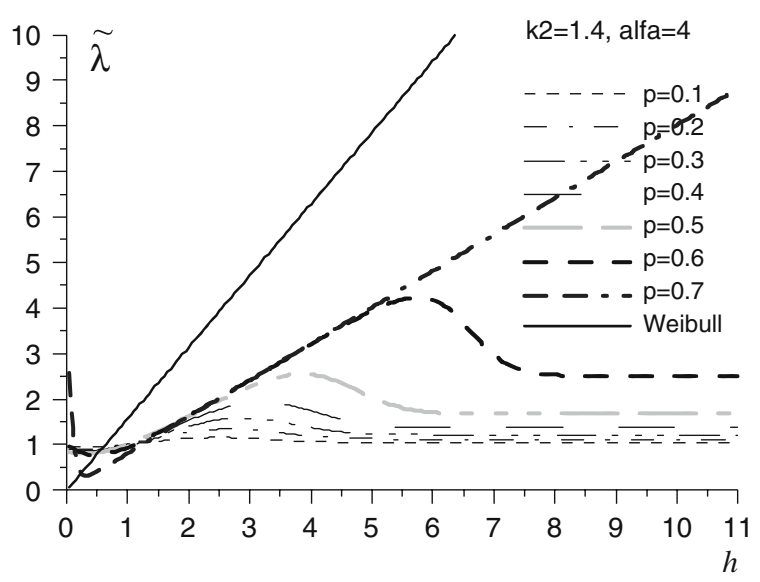

Fig. 2 Dimensionless HR for conjectural "truths" with $k_{2}=1.4$ and $\alpha=4$ 


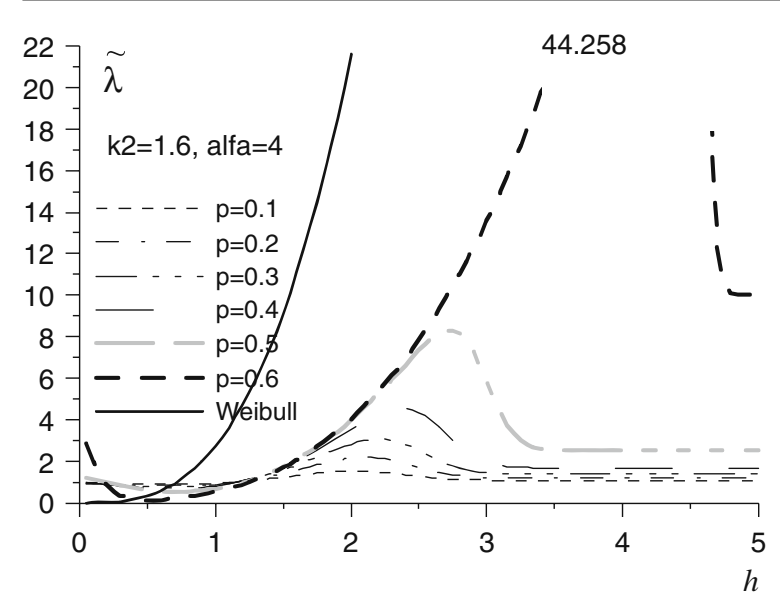

Fig. 3 Dimensionless HR for conjectural "truths" with $k_{2}=1.6$ and $\alpha=4$

Let us remember that all distributions (Eq. 11) have mean value $=1$. If we want the prediction in time scale, we will use the relationships:

$\tilde{\lambda}(h)=\mu \lambda\left(t_{0}\right) ; P_{\Delta t \mid t_{0}}=P_{\Delta h \mid h}$ with $\Delta t=\mu \Delta h$,

$\tilde{F}(h)=F_{\tau}\left(t_{0}\right)$ and $\tilde{f}(h)=\mu f_{\tau}\left(t_{0}\right)$.

Moreover, the asymptotic value of the hazard rate is given by $\lambda_{\infty}=1 / \mu_{1}$ and its dimensionless form is given by $\tilde{\lambda}_{\infty}=1 / k_{1}$.

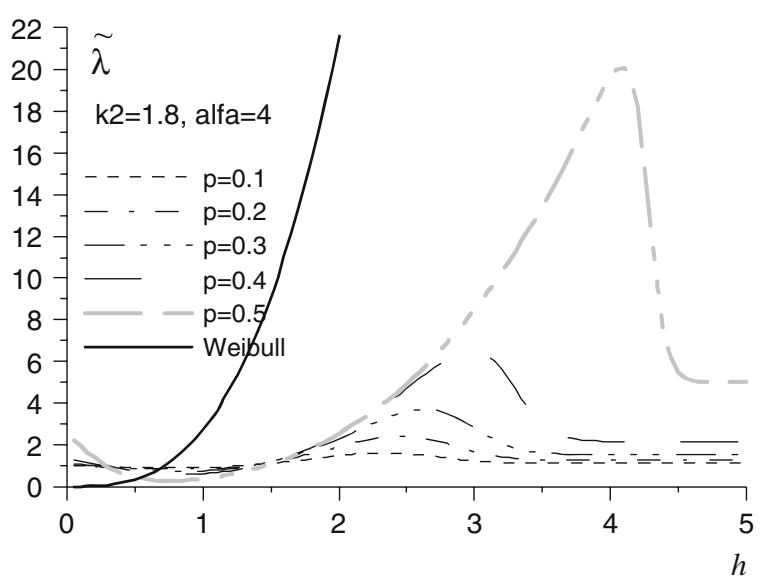

Fig. 4 Dimensionless HR for conjectural "truths" with $k_{2}=1.8$ and $\alpha=4$

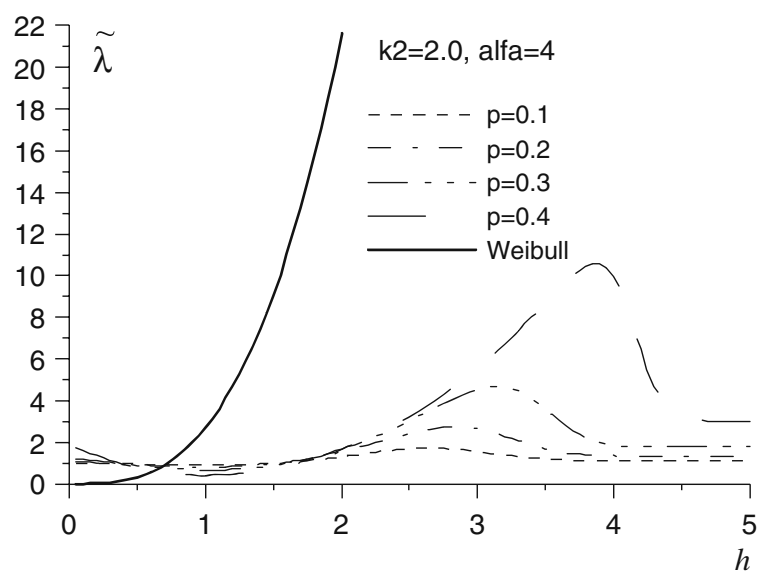

Fig. 5 Dimensionless HR for conjectural "truths" with $k_{2}=2.0$ and $\alpha=4$

\section{Comparison between estimation procedures}

We consider four different estimation procedures for conditional earthquake occurrence probability.

The first one assumes the mathematical model (Eq. 11) and it uses the classical method of maximum likelihood (ML) for the estimation of the parameters $k_{1}$ and $k_{2}$ (Maybeck 1979).

A second method, proposed by us and called the threshold method (TR), assumes again the mathematical model (Eq. 11) with the means of interoccurrence times less than and greater than 1 taken as estimators of $k_{1}$ and $k_{2}$, respectively. As we will see in the following, if $r$ assumes values around 4 or more, the TR method is better than

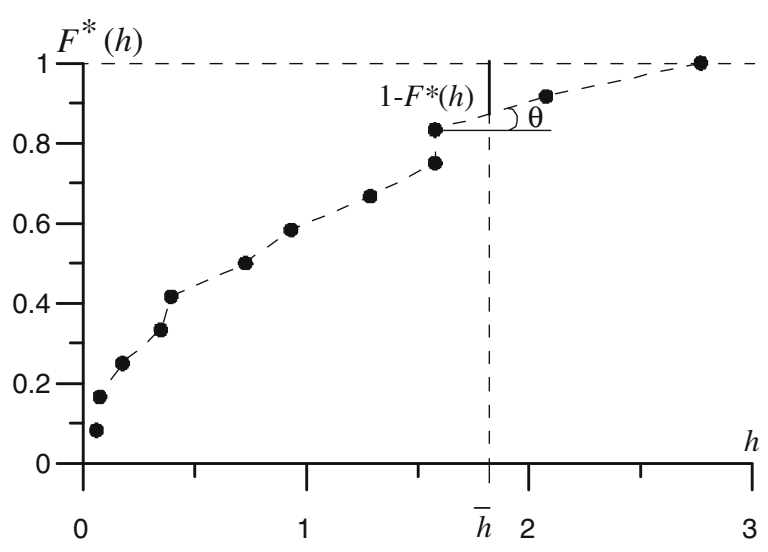

Fig. 6 Building of empirical value $\lambda^{*}$ as exposed in Eq. 13 
the ML method: indeed, it is able to catch the contribution of the few interoccurrence times in the tail better than the ML, which is sensitive to the total contribution of the interoccurrence times. On the other hand, the TR fails for low values of $r$, i.e. if the tails of the two components of the mixture overlap.

A third method (ME) is based on the maximum entropy principle that implies the use of a generalised exponential distribution (Jaynes 1957; Akaike 1977; Tagliani 1989, 1990). Indeed, this is the distribution function that incorporates only the information inherent in dataset (so maximising the entropy of the distribution). This method does not agree with our choice of limited $\lambda_{\infty}$, but it is useful in a qualitative sense because it is very sensitive to the dataset and is free from the characteristic earthquake or other interpreting hypothesis (Vere-Jones and Ozaki 1982).

Finally, an empirical method (EMP) consists in reading directly on the cumulative frequency polygon $F^{*}$ the values of $\lambda^{*}$ and $P_{\Delta h \mid h}$. Specifically, the empirical value $\lambda *$ is:

$\lambda^{*}(h)=\frac{(\operatorname{tg} \theta)_{h}}{1-F^{*}(h)}$

being $(\operatorname{tg} \theta)_{h}$ the tangent of that polygon $F^{*}$ side which $h$ belongs to (Fig. 6). Equation 16 becomes meaningless at the end of the polygon, but, before the end, it offers an empirical rough prediction directly coming from the dataset, free from mathematical model (apart the piecewise linearity of the polygon). Moreover, the dataset can suggest possible variability of the hazard that the structure of the chosen mathematical model cannot incorporate; typically, a possible multimodality of the hazard will be suggested from the empirical method. Obviously, this rough empirical method EMP cannot be considered as a reliable nonparametric procedure, but it may furnish complementary qualitative information.

\section{Procedure credibility}

In previous papers (Grandori et al. 1998, 2003, 2006; Guagenti et al. 2003, 2004), the credibility of a procedure has been introduced: a measure of the error, with respect to a conjectural truth $F^{\circ}$, when a target quantity $A$ is estimated with that procedure. Let $A^{\circ}$ be the true value of $A$ (in the conjectural truth) and $\hat{A}_{s}$ its estimator with the procedure $s$. The credibility $\Delta_{s}^{\circ}$ of the procedure $s$ relative to the truth $F^{\circ}$ (that here is simply a given distribution) is defined as follows:

$\Delta_{s}^{\circ}=\operatorname{Pr}\left\{A^{\circ}-k A^{\circ} \leq \hat{A}_{s} \leq A^{\circ}+k A^{\circ}\right\}$.

In other words, $\Delta_{s}^{\circ}$ is the probability to have (as absolute value) a fractional (or relative) error not larger than a given value $k$ in the estimate of the quantity $A$. In this research, the target quantity $A$ is the prediction $\left(\lambda\right.$ or $\left.P_{\Delta t \mid t_{0}}\right)$.

The merit of credibility $\Delta_{S}^{\circ}$ is to focus attention on the target quantity $A$ of interest, instead of on the data fitting. This makes the credibility specifically useful to the aim of the research and more selective than classic tests. For example, exponential, lognormal, gamma and Weibull distributions, largely used for interoccurrence time model, can lead, with the classic tests, to similar levels of significance; on the other hand, they lead to predictions drastically different.

It is remarkable that the credibility $\Delta_{S}^{\circ}$ is not based on the single sample given by the catalogue but on "all samples" (i.e. 1,000 samples) that can be drawn from $F^{\circ}$.

Because of definition (17) of credibility, competing models can be compared in the frame of the chosen conjectural truths. The values of $\Delta$ are obtained with Monte Carlo simulation.

In Tables 1 and 2 , the $\Delta$ values are shown, relative to the estimated quantity $\hat{\lambda}$ or $\hat{P}$ in respect to some conjectural $F^{\circ}$ identified through their single parameter $k_{2}^{\circ}$, being the comparison done with the same values of $p$ and $\alpha$. The values $p=0.5$ and $\alpha=4$ are assumed because they are frequently observed in the studied zones; the parameter $k_{1}^{\circ}$ is evaluated through Eq. 12.

Table 1 gives the credibilities of the estimate of $\tilde{\lambda}$ with the procedures ML and TR. Table 2 gives the same credibilities for the estimate of $P_{\Delta h \mid h}$. The sample size is rather numerous in this first analysis: it is $v=100$. The threshold $k$ is taken as equal to 0.3 ; a series of preliminary tests in various hypothetical conditions confirmed a good sensitivity of the method of comparison based on $k=0.3$. 
Table 1 Values of $\Delta=$ $\operatorname{Pr}\left\{\left|\frac{\hat{\lambda}(h)-\lambda^{\circ}(h)}{\lambda^{\circ}(h)}\right|<0.3\right\}$ with samples size $v=100$

\begin{tabular}{lllllccc}
\hline$h$ & Methods & 0.5 & 1 & 1.5 & 2 & 2.5 & $h_{\max }$ \\
\hline$k_{2}^{\circ}$ & & & & & & & \\
\hline 1.2 & ML & 0.99 & 0.89 & 0.94 & 0.49 & 0.87 & 0.890 \\
& TR & 1.00 & 0.02 & 0.22 & $\sim 0.0$ & $\sim 0.0$ & $\sim 0.0$ \\
1.4 & ML & 0.96 & 0.93 & 0.81 & 0.74 & 0.45 & 0.50 \\
& TR & 1.00 & 0.76 & 0.67 & 0.93 & $\sim 0.0$ & 0.09 \\
1.6 & ML & 0.92 & 0.93 & 0.76 & 0.73 & 0.74 & 0.82 \\
& TR & 1.00 & 0.94 & 0.84 & 0.83 & 0.88 & 0.87 \\
1.8 & ML & 0.61 & 0.80 & 0.65 & 0.64 & 0.64 & 0.92 \\
& TR & 0.57 & 0.83 & 0.71 & 0.71 & 0.71 & 0.80 \\
\hline
\end{tabular}

Table 2 Values of $\Delta=$ $\operatorname{Pr}\left\{\left|\frac{\hat{P}(h)-P^{\circ}(h)}{P^{\circ}(h)}\right|<0.3\right\}$; $\Delta h=0.1$ and with samples size $v=100$

\begin{tabular}{rllllrrr}
\hline \multicolumn{1}{l}{$\boldsymbol{h}$} & Methods & 0.5 & 1 & 1.5 & 2 & 2.5 & $h_{\max }$ \\
\hline$k_{2}$ & & & & & & & \\
\hline 1.2 & ML & 0.99 & 0.91 & 0.96 & 0.59 & 0.89 & 0.90 \\
& TR & 1.00 & 0.03 & 0.51 & $\sim 0.00$ & $\sim 0.00$ & 0.01 \\
1.4 & ML & 0.97 & 0.93 & 0.86 & 0.80 & 0.53 & 0.53 \\
& TR & 1.00 & 0.75 & 0.75 & 0.98 & $\sim 0.00$ & 0.11 \\
1.6 & ML & 0.92 & 0.91 & 0.79 & 0.84 & 0.85 & 0.88 \\
& TR & 1.00 & 0.93 & 0.87 & 0.90 & 0.95 & 0.97 \\
1.8 & ML & 0.61 & 0.77 & 0.68 & 0.71 & 0.75 & 0.98 \\
& TR & 0.54 & 0.82 & 0.73 & 0.75 & 0.80 & 0.94 \\
\hline
\end{tabular}

Table 3 Values of $\Delta=$ $\operatorname{Pr}\left\{\left|\frac{\hat{\lambda}(h)-\lambda^{\circ}(h)}{\lambda^{\circ}(h)}\right|<0.3\right\}$ with samples size $v=20$

\begin{tabular}{llllcccc}
\hline \multicolumn{1}{l}{} & Methods & 0.5 & 1 & 1.5 & 2 & 2.5 & $h_{\max }$ \\
\hline$k_{2}^{\circ}$ & & & & & & & \\
\hline 1.2 & ML & 0.71 & 0.57 & 0.57 & 0.19 & 0.50 & 0.49 \\
& TR & 0.91 & 0.21 & 0.37 & $\sim 0.0$ & $\sim 0.0$ & $\sim 0.0$ \\
1.4 & ML & 0.67 & 0.61 & 0.46 & 0.38 & 0.25 & 0.36 \\
& TR & 0.91 & 0.65 & 0.54 & 0.68 & 0.14 & 0.70 \\
1.6 & ML & 0.58 & 0.59 & 0.41 & 0.39 & 0.34 & 0.69 \\
& TR & 0.87 & 0.70 & 0.54 & 0.54 & 0.58 & 0.69 \\
1.8 & ML & 0.33 & 0.41 & 0.36 & 0.34 & 0.34 & 0.43 \\
& TR & 0.38 & 0.49 & 0.38 & 0.38 & 0.40 & 0.57 \\
\hline
\end{tabular}

Table 4 Values of $\Delta=$ $\operatorname{Pr}\left\{\left|\frac{\hat{P}(h)-P^{\circ}(h)}{P^{\circ}(h)}\right|<0.3\right\}$; $\Delta h=0.1$ and with samples size $v=20$

\begin{tabular}{lllllccr}
\hline$h$ & Methods & 0.5 & 1 & 1.5 & 2 & 2.5 & $h_{\max }$ \\
\hline$k_{2}$ & & & & & & & \\
\hline 1.2 & ML & 0.78 & 0.58 & 0.59 & 0.24 & 0.51 & 0.49 \\
& TR & 0.90 & 0.20 & 0.37 & $\sim 0.0$ & $\sim 0.0$ & $\sim 0.0$ \\
1.4 & ML & 0.70 & 0.58 & 0.53 & 0.45 & 0.28 & 0.28 \\
& TR & 0.92 & 0.63 & 0.55 & 0.68 & 0.14 & 0.70 \\
1.6 & ML & 0.58 & 0.58 & 0.44 & 0.48 & 0.48 & 0.77 \\
& TR & 0.88 & 0.69 & 0.50 & 0.53 & 0.57 & 0.63 \\
1.8 & ML & 0.32 & 0.41 & 0.34 & 0.34 & 0.38 & 0.79 \\
& TR & 0.41 & 0.50 & 0.36 & 0.35 & 0.36 & 0.59 \\
\hline
\end{tabular}




\begin{tabular}{|c|c|c|c|c|c|c|c|c|}
\hline \multirow[t]{16}{*}{$\begin{array}{l}\text { Table } 5 \text { Values of } \\
\Delta_{\delta}^{\prime}=\operatorname{Pr}\{\hat{\lambda}(h)-1>2\} \\
\text { with samples size } v=20\end{array}$} & $\frac{h}{k_{2}}$ & Methods & 0.5 & 1 & 1.5 & 2 & 2.5 & $h_{\max }$ \\
\hline & $\overline{1.2}$ & ML & 0.00 & 0.00 & 0.42 & 0.46 & 0.25 & 0.47 \\
\hline & & $\mathrm{TR}$ & 0.00 & 0.00 & $\sim 0.0$ & 0.79 & 0.68 & 0.71 \\
\hline & & $\mathrm{ME}$ & 0.01 & 0.08 & 0.28 & 0.21 & 0.24 & 0.15 \\
\hline & 1.4 & ML & 0.00 & 0.00 & 0.29 & 0.67 & 0.54 & 0.77 \\
\hline & & $\mathrm{TR}$ & 0.00 & 0.00 & $\sim 0.0$ & 0.74 & 0.84 & 0.88 \\
\hline & & $\mathrm{ME}$ & 0.02 & 0.04 & $\sim 0.0$ & 0.50 & 0.64 & 0.99 \\
\hline & 1.6 & ML & 0.00 & 0.00 & 0.12 & 0.54 & 0.74 & 0.87 \\
\hline & & TR & 0.00 & 0.00 & $\sim 0.0$ & 0.45 & 0.84 & 0.88 \\
\hline & & $\mathrm{ME}$ & 0.02 & 0.01 & 0.09 & 0.35 & 0.81 & 0.99 \\
\hline & 1.8 & ML & 0.00 & 0.00 & $\sim 0.0$ & 0.32 & 0.64 & 0.86 \\
\hline & & TR & 0.00 & 0.00 & $\sim 0.0$ & 0.21 & 0.58 & 0.76 \\
\hline & & ME & 0.01 & 0.01 & 0.04 & 0.18 & 0.50 & 1.00 \\
\hline & $0(\exp )$ & ML & 0.00 & 0.00 & 0.00 & 0.00 & 0.00 & 0.00 \\
\hline & & TR & 0.00 & 0.00 & 0.00 & 0.00 & 0.00 & 0.00 \\
\hline & & ME & 0.05 & 0.04 & 0.04 & 0.05 & 0.04 & 0.00 \\
\hline
\end{tabular}

The credibilities are time-dependent; hence, their values are calculated for different time instant $h$ with steps of 0.5 , i.e. 0.5 times the return period $\mu$. These step values allow determining in which periods the prediction is more or less reliable. The last column gives the mean value of the credibilities if the prediction is made when the elapsed time $h$ is equal to the maximum observed interoccurrence times.

In some cases, the tables show large differences. Which one must be judged more credible? On the basis of many numerical simulations, we can say that the TR method is reliable when the ratio $r=k_{2} / k_{1}$ is large $(r>4)$, as in the case of the third or fourth row. This behaviour can be explained thanks to the meaning of the ratio $r$ : its large values (third and fourth cases) mean a weak overlap of the tails of the two component densities (ex and w) and the information coming from the larger IT is really interpreted as Weibull contribution, whilst small values of $r$ mean that the role of the two components is not clearly separate in the interpretation of short and long interoccurrence times.

The seismic zones in this study have much fewer data than in this first simulation. So, Tables 3 and 4 give the credibilities when the sample has the more realistic size of 20 elements $(v=20)$. The credibility values are obviously rather small, but they confirm the preceding trend.

With regard to the ME method, its credibility is almost always around 0.25 : a reliable prediction cannot be based on it. Nevertheless, as already noticed, from a qualitative point of view, it offers useful information directly coming from data, free of modelling error; therefore, it must be taken into consideration, especially when it appears largely different from the assumed model.

All the methods are significantly reliable in deciding whether the Poissonian model could be rejected. Indeed, in case of the Poisson model, $\mathrm{HR}=1$. If the credibility is aimed at testing the hypothesis $\mathrm{HR}=$ constant against any other time varying $H R$, it becomes:

$\Delta_{\delta}=\operatorname{Pr}\left\{\hat{\lambda}(h)-1<k_{\delta}\right\}$.

Equation 18 is the probability of an error not larger than a given value $k_{\delta}$ accepting the hypothesis HR = constant. Table 5 gives its complementary values $\Delta_{\delta}^{\prime}$ with $k_{\delta}=2$. Therefore, the table shows the credibility of an HR more than three times the Poissonian constant HR. All the three methods, even if the probability can be different, agree in judging the difference from the Poisson hypothesis, when it exists.

Table 6 Values of $\Delta_{\mathrm{ex}-\mathrm{w}}^{\exp }$ for estimate with ML of $\tilde{\lambda}$ and $P_{0.1 \mid h} ;$ with samples size $v=20$

\begin{tabular}{lllllll}
\hline$h$ & 0.5 & 1 & 1.5 & 2 & 2.5 & $h_{\max }$ \\
\hline$\tilde{\lambda}$ & 0.92 & 0.95 & 0.70 & 0.57 & 0.48 & 0.42 \\
$P_{0.1 \mid h}$ & 0.94 & 0.95 & 0.71 & 0.59 & 0.50 & 0.44 \\
\hline
\end{tabular}


Table 7 Building of a MR interoccurrence time dataset using the interoccurrence time datasets of the constituent SA's

\section{Robustness}

The credibility procedure is now applied to investigate the robustness of the mixture procedure. In particular, assumed as true distribution of the interoccurrence times the exponential process, we drew from it several random samples using the Monte Carlo method. Considered as competing models, the ex-w mixture distribution and the exponential distribution, from each sample, we derived, with the ML method, the model parameters and we evaluated the hazard rate curves $\tilde{\lambda}$. Finally, we calculated the credibilities (in $\tilde{\lambda}$ and $P_{0.1 \mid h}$ estimate) of the mixture procedure, $\Delta_{\mathrm{ex}-\mathrm{w}}^{\exp }$, i.e. when the mixture is a wrong model being the truth exponential. From the obtained results, it is evident that the ex-w mixture model has the advantage of being robust, at least in respect to the Poissonian hypothesis. Indeed, the mixture model gives a reliable prediction even if the true distribution is exponential, as shown in Table 6 , because of its adaptability. Note that the credibility values are rather high, even if a realistically small size of the samples $(v=20)$ was chosen.
Moreover, it was verified that the above credibility $\Delta_{\mathrm{ex}-\mathrm{w}}^{\exp }$ is about of the same order of the credibility $\Delta_{\exp }^{\exp }$ it means that the degree of uncertainty of the wrong (but robust) mixture procedure is similar to the degree of uncertainty due to statistical variability of the samples in the true exponential model.

Repeating the same procedure assuming the exp-w mixture process as true distribution, we verified the lack of robustness of the exponential model. In fact its credibility, when the truth is a mixture, is near to zero.

\section{Application to Italian seismic sources}

The catalogues taken into consideration for the application of the proposed procedures are obtained by the association of the events of the Italian historic catalogue CPTI04 (CPTI Working Group 2004) with the seismogenetic source areas (SA) defined in DISS3.0.2 (Database of Individual Seismogenetic Sources [DISS]; DISS Working

Table 8 MR6: seismic catalogue in dimensional and dimensionless form

\begin{tabular}{|c|c|c|c|c|c|c|c|}
\hline \multicolumn{8}{|c|}{ MR6_interoccurrence times in years } \\
\hline 4.688642 & 5.881749 & 13.76984 & 27.18319 & \multirow{2}{*}{30.71288} & \multirow[t]{2}{*}{56.73634} & \multirow[t]{2}{*}{72.65607} & \multirow[t]{2}{*}{100.3346} \\
\hline 122.9360 & 122.937 & 161.8029 & 215.7441 & & & & \\
\hline \multicolumn{8}{|c|}{$\mu=77.94861, p=0.4167, \lambda_{\infty}=0.033077, \tilde{\lambda}_{\infty}=2.5783$} \\
\hline \multicolumn{8}{|c|}{ MR6_interoccurrence times in dimensionless form } \\
\hline 0.060150 & 0.075457 & 0.176653 & 0.348732 & 0.394014 & 0.727869 & 0.932102 & 1.287189 \\
\hline 1.577142 & 1.577154 & 2.075764 & 2.767774 & & & & \\
\hline
\end{tabular}



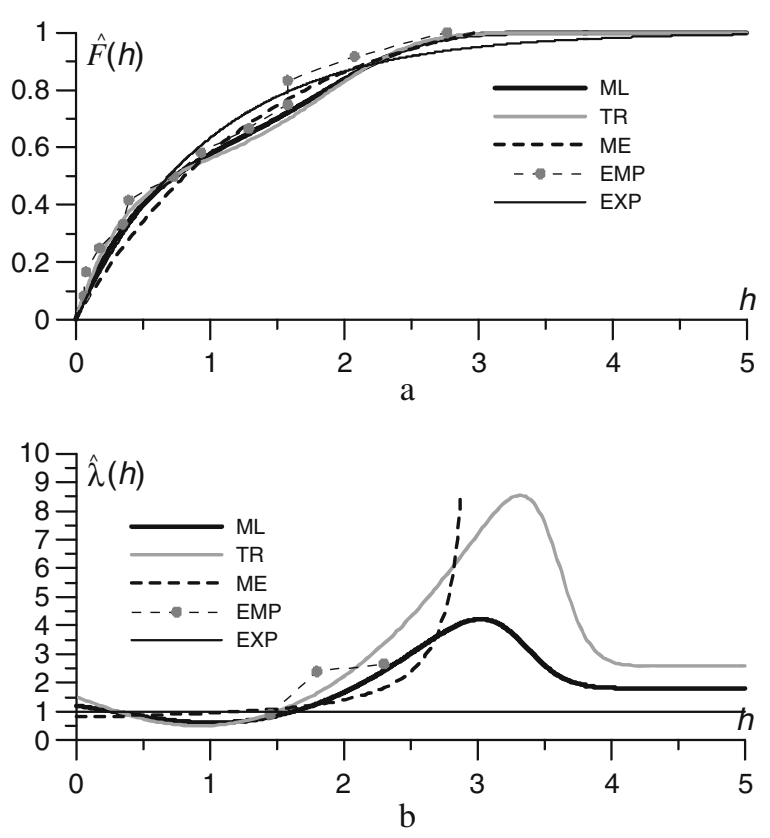

Fig. 7 (a) MR6: estimation of $\hat{F}(h)$ in dimensionless form. (b) MR6: estimation of $\hat{\lambda}(h)$ in dimensionless form

Group 2006). In DISS, SAs are identified on the basis of geological and geophysical characteristics (Basili 2007).

These new sub-catalogues have been made available by the members of Task 1.1 of the INGV-DPC Project S2 (Slejko and Valensise coord.). Each analysed catalogue involves events with magnitude $M$ defined in CTPI04 as Maw $>5.3$ and having occurred from 1600 to 2002. To better understand the results presented below, we have provided some supplementary electronic material (the label "SEM", included in table and figure references, will be used to identify further supplementary electronic material associated with this paper). In Figure 01SEM of the Supplementary Electronic Material, a plot of the SA's labelled with the identification number used in DISS3.0.2 is given.

As explained in the previous sections, the sample size $v$ plays an important role on the reliability of a probabilistic analysis. Initial inspection of the catalogues of the 74 SA Sources reveals immediately that the methods of this paper cannot be applied successfully: many of the SA sources have too small a sample size $v$ (zero to five events and zero to four interoccurrence times). We obtained a set of sources having a sufficiently large set of interoccurrence times, using the new association of source areas and related seismicity, grouping the SA's into eight macro regions (MR), on the basis of geophysical aspects (Basili 2007). These are shown in Figure 02SEM of the Supplementary Electronic Material; the MR coordinates are listed in Table 01SEM of the Supplementary Electronic Material.

The combining of SAs in MR can lead to lose the proper identity of each SA. To maintain unaltered the characteristics of the interoccurrence

Table 9 MR6: probability of occurrence $\hat{P}_{\Delta t \mid t_{0}}$ for different $\Delta t$ and different approach of each SA

\begin{tabular}{|c|c|c|c|c|c|c|}
\hline MR6 & $\Delta t=5$ years & $\Delta t=10$ years & $\Delta t=20$ years & $\Delta t=30$ years & $\Delta t=50$ years & $\Delta t=100$ years \\
\hline \multicolumn{7}{|c|}{$\mathrm{SA} 24, t_{0}=40$} \\
\hline ML & 0.039451 & 0.096306 & 0.175295 & 0.237261 & 0.351327 & 0.627267 \\
\hline TR & 0.04145 & 0.088838 & 0.147305 & 0.195926 & 0.281847 & 0.580433 \\
\hline $\mathrm{ME}$ & 0.042291 & 0.106876 & 0.204251 & 0.286431 & 0.44201 & 0.721479 \\
\hline \multicolumn{7}{|c|}{$\mathrm{SA} 34, t_{0}=22$} \\
\hline ML & 0.056713 & 0.107703 & 0.207961 & 0.277258 & 0.392574 & 0.612508 \\
\hline TR & 0.058281 & 0.108403 & 0.200431 & 0.259055 & 0.350627 & 0.559674 \\
\hline $\mathrm{ME}$ & 0.049064 & 0.096063 & 0.198182 & 0.277916 & 0.428859 & 0.700014 \\
\hline \multicolumn{7}{|c|}{$\mathrm{SA} 38, t_{0}=4$} \\
\hline ML & 0.06687 & 0.136225 & 0.243931 & 0.323475 & 0.445231 & 0.636549 \\
\hline TR & 0.080004 & 0.148542 & 0.272828 & 0.349374 & 0.456243 & 0.607427 \\
\hline $\mathrm{ME}$ & 0.047855 & 0.101151 & 0.193301 & 0.271068 & 0.413136 & 0.682776 \\
\hline \multicolumn{7}{|c|}{$\mathrm{SA} 63, t_{0}=92$} \\
\hline ML & 0.038474 & 0.083398 & 0.16832 & 0.249702 & 0.435952 & 0.845912 \\
\hline TR & 0.032162 & 0.078694 & 0.150611 & 0.231663 & 0.429369 & 0.867464 \\
\hline ME & 0.057749 & 0.122077 & 0.233283 & 0.327149 & 0.504588 & 0.823986 \\
\hline
\end{tabular}




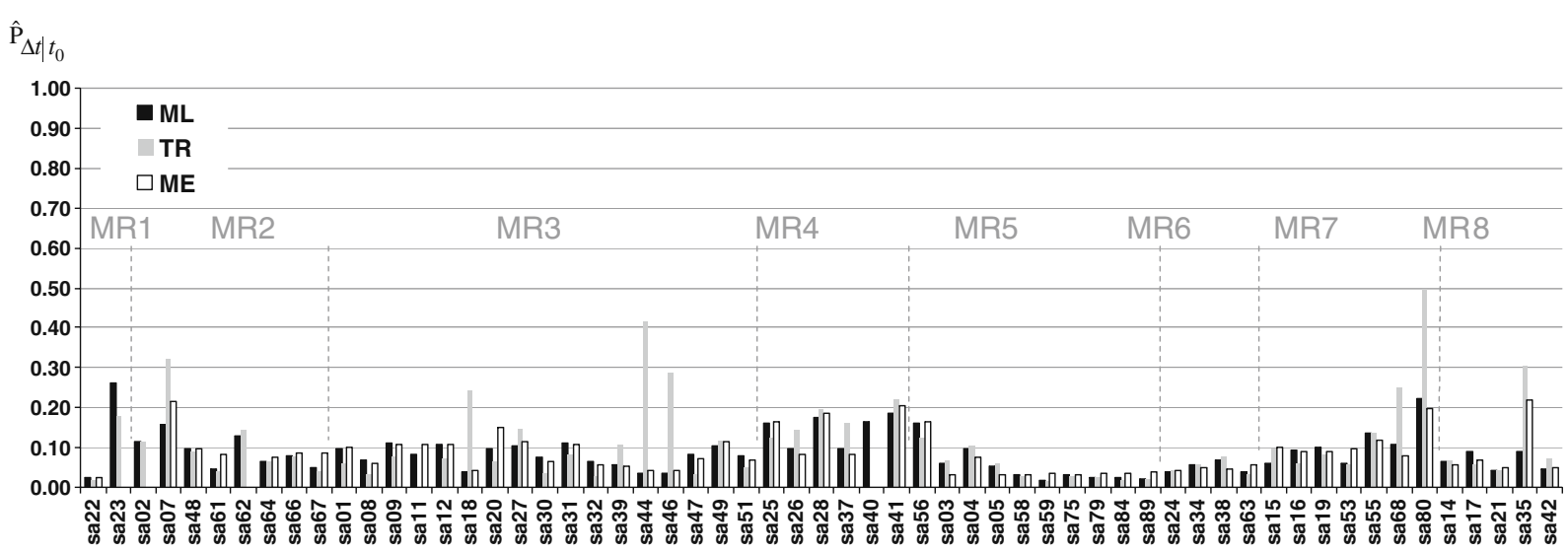

Fig. 8 Italian SA sources: conditional occurrence probability for $\Delta t=5$ years, given elapsed time $t_{0}$, different for each SA. Comparison between the ML, TR and ME methods. The vertical dotted lines define the eight MR

of each SA, the interoccurrence times of each MR are built using the interoccurrence times obtained for each SA; as an example, Table 7 shows the data set of MR6 built using the data set of SA24, SA34, SA38 and SA63. The implicit assumption is that the constituent sources in a region behave similarly in their seismic cycles. The choices made during this phase of the project were discussed and decided with other components of the INGVDPC Project S2 with the aim that the results obtained by the tasks involved into the project could be comparable each other.

The three methods, ML, TR and ME, proposed in the previous sections were applied to the eight Italian MR. For each MR, the data were organised as shown in Table 8 and Fig. 7 for MR6. In Table 8 are collected the MR6 catalogue in dimensional and dimensionless forms, the MR6 return period $\mu$, the parameter $p$, representative of the evidence of characteristic earthquake, the asymptotic hazard rate value $\lambda_{\infty}$ and its dimensionless form $\tilde{\lambda}_{\infty}$. Figure 7a, b show, respectively, the MR6 $\hat{F}(h)$ and $\hat{\lambda}(h)$ estimations with the three methods in dimensionless form. In the figure, the experimental HR $\lambda^{*}$ (EMP) and the exponential HR (EXP) are also presented. The behaviour of EMP is useful for investigating anomalies in the data sets not captured by ML, TR and ME.

All the results produced are collected in the SEM file of this paper (Figures 03SEM-10SEM and Tables 02SEM-09SAM of the Supplementary Electronic Material).

In all the implementations, the exponential distribution seems inadequate to model the seismic

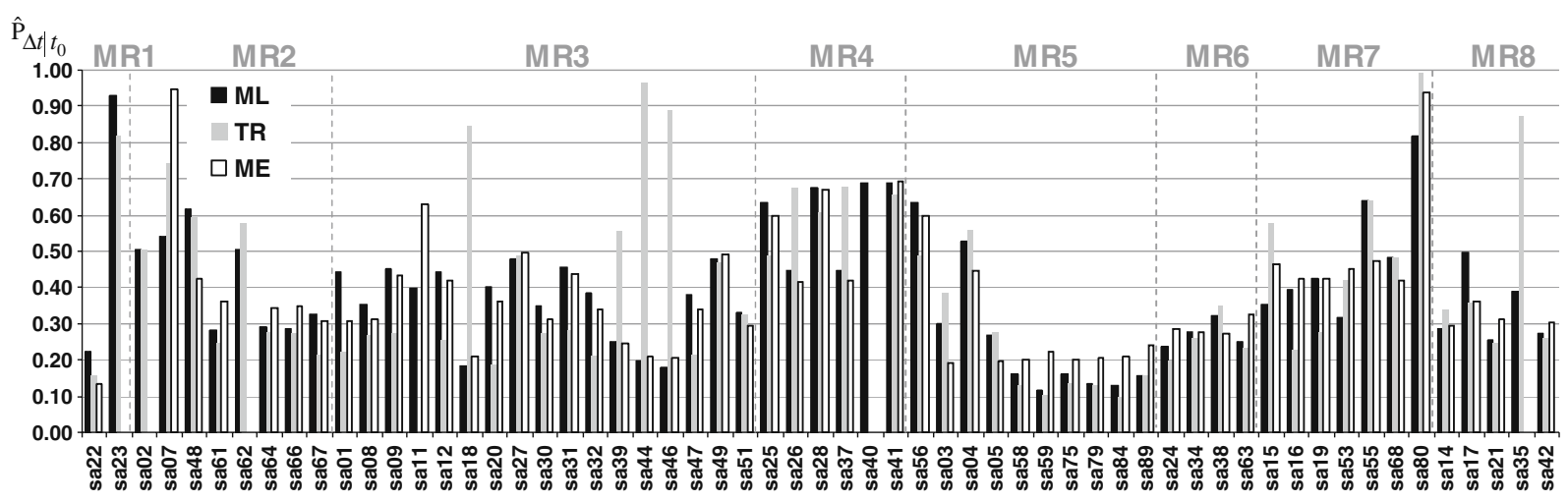

Fig. 9 Italian SA sources: conditional occurrence probability for $\Delta t=30$ years, given elapsed time $t_{0}$, different for each SA. Comparison between the ML, TR and ME methods. The vertical dotted lines define the eight MR 


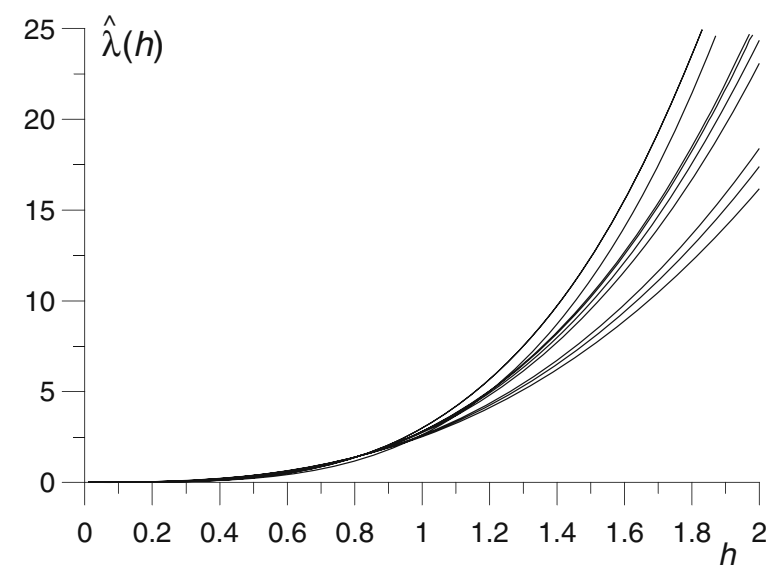

Fig. 10 Mixture-estimated $\hat{\lambda}(h)$ (in dimensionless form) for samples drawn from known distribution with increasing hazard rate (Weibull with $\alpha>1$ )

renewal process, whilst the mixture ex-w, supported by the proposed credibility analysis, seems more adequate.

The next step was the evaluation of the probability $\hat{P}_{\Delta t \mid t_{0}}$ : it is the probability of occurrence of an event of magnitude Maw $>5.3$ (according with the events considered in the project) in the next interval of time $\Delta t$ if a time $t_{0}$ is passed from the last event occurring in each SA and the last year reported in CPTI04 (2002) assumed as date of reference. Different intervals of time $\Delta t$ have been considered: 5, 10, 20, 3050 and 100 years. In particular, the probability obtained for $\Delta t$ equal 5 and 30 years are collected, both in tables and in maps, in Figures 11SEM-16SEM and Tables 01_PSEM-08P_SEM of the Supplementary Electronic Material of this paper. As example, Table 9 shows the probability of occurrence connected with the SA classified in MR6.

Since the year 2002 (the end of our catalogue) until today, 5 years have already passed. The occurrence probabilities shown in Table 9 are low and the earthquake did not occur. Is this a confirmation of the model goodness? Not at all. It can only be taken as a kind of consolation. The degree of goodness of the model is measured by the above shown credibility that had explored the "complete" (1,000 samples) sequence of possible events.

Figures 8 and 9 collect and compare the probability obtained in each SA with the three differ- ent methods proposed for $\Delta t=5$ years and $\Delta t=$ 30 years (in the Supplementary Electronic Material file, Figs. 8 and 9 are reported in larger format and labelled as Figure 17SEM and Figure 18SEM, respectively). We may observe that, in most of the cases, the three methods give comparable results. There are few cases in which the ML and ME methods produce lower probabilities than the TR method. It could be due to the frame of the TR method more able to take into account the tail of the distribution when the ratio $r$ is large, as previously discussed: accordingly, the high value of TR probability may indicate peaks in the hazard curve not caught by ML and ME methods. In any case, these situations should be investigated. However, when the gap $t_{0}$ is large (i.e. SA40 and SA11), the TR prediction cannot be done because the asymptotic behaviour of the hazard rate has been reached. ME results mainly confirm the results of ML. On the other hand, being ME method strictly related to datasets, in few cases it gives differences or even lack of prediction. Considering ML results for $\Delta t=5$ years, the highest hazard of occurrence are found in SA 23 (MR1-Western Alps), in SA 80 (MR7-Calabrian Arc) and in the SA's of the Central Northern Apennines (MR4). Increasing the interval of time up to 30 years, the hazard increases maintaining mainly the same ratio between the different areas. It indicates that not significant changes should be expected in the

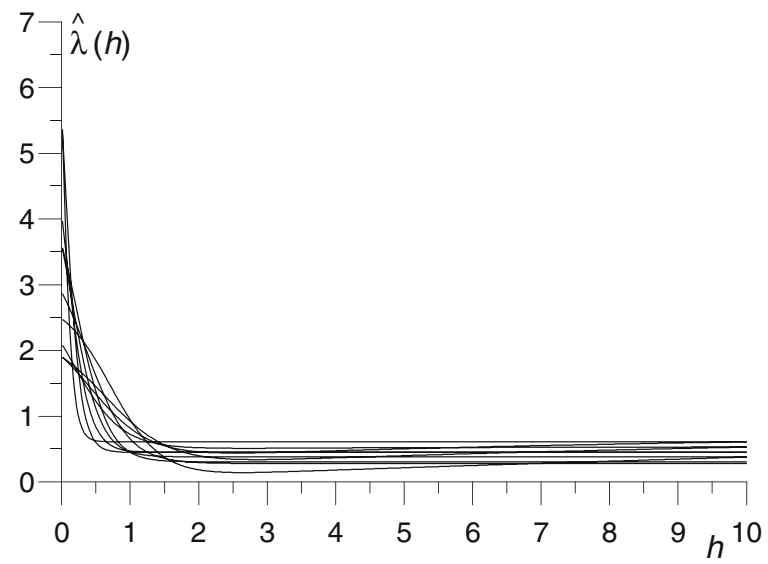

Fig. 11 Mixture-estimated $\hat{\lambda}(h)$ (in dimensionless form) for samples drawn from known distribution with decreasing hazard rate (Weibull with $\alpha<1$ ) 
hazard of the country increasing the expected time from 5 to 30 years, according to the ML and ME methods. On the contrary, TR method underlines some differences.

\section{Discussion and conclusions}

Within the context of earthquake prediction, if it is admissible to assume that the generation of strong events depends primarily on the time elapsed since the last event, at least in some seismic areas, and if, in this reduced context, it is reasonable to accept an asymptotic stationary prediction, an exponential-Weibull mixture renewal model, associated with an empirical criterion, leads to conditional probabilities of occurrence having a rather good degree of credibility, at least for the Italian sources considered.

The proposed model appears flexible enough to interpret different behaviours of earthquake occurrence, whose identification is up to this day questionable. It is a robust model; in particular, it can give a statistic symptom of the dominant behaviour in the analysed regions: clustering, seismic gap or Poissonian one.

The shape parameter $\alpha$ of the Weibull component is responsible for the increasing or decreasing hazard rate; the hazard rate being nearly constant when $\alpha$ is near to 1 .

It is remarkable that the proposed mixture spontaneously fits the alternative behaviours with a good credibility. As an example, let us consider samples drawn from two known distributions with increasing or decreasing hazard rate, respectively; precisely two Weibull distributions, respectively with $\alpha>1$ and $\alpha<1$ are assumed as conjectural truths. Figures 10 and 11 show the estimated hazard rate with the ex-w mixture model. It is evident that, even if the mixture is a wrong model in this case, it is able to catch the true increasing or decreasing behaviour of the hazard rate in the two assumed conjectural truths.

In the regions analysed in this paper, the model leads to estimate values of $\alpha>1$. On the other hand, we have to stress that the robustness of the mixture model refers only to the explored distributions $F^{\circ}$. The panorama of conjectural truths $F^{\circ}$ and the target quantities $A$ should be properly enriched; other models can enter in competition. Nevertheless, all the numerical experiments carried out up to now showed a remarkable robustness of the model, i.e. its adoption reduces the epistemic uncertainties.

The credibility $\Delta_{s}^{\circ}$ can be a good tool to judge a model in its structure in a panorama of conjectural truths, rather than to check a hypothesis upon a single catalogue.

Acknowledgements This research has been developed in the frame of the Project S2-Assessing the seismogenetic potential and the probability of strong earthquakes in Italy (Slejko and Valensise coord.) - S2 Project has benefited from funding provided by the Italian Presidenza del Consiglio dei Ministri-Dipartimento della Protezione Civile (DPC). Scientific paper funded by DPC do not represent its official opinion and policies. The authors would also like to deeply thank both reviewers for their valuable comments and suggestions in relation to the shortcomings of the initially submitted work.

\section{References}

Akaike H (1977) On entropy maximization principle. In: Krishnaiah PR (ed) Proceedings of the symposium on application of statistics. Amsterdam, The Netherlands, pp $27-47$

Basili R (2007) Organizzazione di un sistema di riferimento unitario per la valutazione della sismogenesi. Final report of Prj S2: valutazione del potenziale sismogenetetico e probabilità di forti terremoti in Italia, UR 1.1 report. Available at http://diss.rm.ingv.it/ sismogenesi/S2_D1.2.html

Basili R, Valensise G, Vannoli P, Burrato P, Fracassi U, Mariano S, Tiberti MM, Boschi E (2008) The database of individual seismogenic sources (DISS), version 3: summarizing 20 years of research on Italy's earthquake geology. Tectonophysics 453:20-43. doi:10.1016/j.tecto.2007.04.014

Corral A (2005) Mixing of rescaled data and Bayesian inference for earthquake recurrence times. Nonlinear Process Geophys 12:89-100

Corral A (2006) Dependence of earthquake recurrence times and independence of magnitudes on seismicity history. Tectonophysics 424:177-193. doi:10.1016/j. tecto.2006.03.035

Cox DR (1962) Renewal theory. Methuen, London, UK

CPTI Working Group (2004) Catalogo Parametrico dei Terremoti Italiani-Versione 2004 (CPTI04) INGV, Bologna. Available at http://emidius.mi.ingv.it/ CPTI04/

DISS Working Group (2006) Database of individual seismogenic sources (DISS), version 3.0.2: a compilation of potential sources for earthquakes larger than 
M 5.5 in Italy and surrounding areas. Available at http://www.ingv.it/DISS/

Faenza L, Marzocchi W, Boschi E (2003) A nonparametric hazard model to characterize the spatiotemporal occurrence of large earthquakes; an application to the Italian catalogue. Geophys $\mathrm{J}$ Int 155:521-531. doi:10.1046/j.1365-246X.2003.02068.x

Garavaglia E, Guagenti E, Petrini L (2007) The earthquake predictability in mixture renewal models. In: Editor Società Italiana di Statistica (ed) Proceedings of Società Italiana di Statistica, SIS intermediate conference 2007 risk and prediction, Venezia, 6-8 June, invited section. CLEUP, Venezia, vol I, pp 361-372

Grandori G, Guagenti E, Tagliani A (1998) A proposal for comparing the reliabilities of alternative seismic hazard models. J Seismol 2:27-35. doi:10.1023/ A:1009779806984

Grandori G, Guagenti E, Tagliani A (2003) Magnitude distribution versus local seismic hazard. Bull Seismol Soc Am 93(3):1091-1098. doi:10.1785/0120010284

Grandori G, Guagenti E, Petrini L (2006) Earthquake catalogues and modelling strategies. A new testing procedure for the comparison between competing models. $\mathrm{J}$ Seismol 10(3):259-269. doi:10.1007/s10950-006-9015-1

Guagenti E, Garavaglia E, Petrini L (2003) Probabilistic hazard models: is it possible a statistical validation? In: Bontempi F (ed) System-based vision for strategic and creative design. Proceedings of ISEC02, 2nd international structural engineering and construction confer- ence, Rome, Italy, U.E., 23-26 September. Balkema, Lisse, The Netherlands, vol II, pp 1211-1216

Guagenti E, Garavaglia E, Petrini L (2004) A proposito di validazione. In: Lagomarsino S, Resemini S (eds) Proceedings of ANIDIS04, XI Conv. Naz. "L'Ingegneria sismica in Italia", Genova, 25-29 January. SGEditoriali, Padova, CD-Rom

Guagenti Grandori E, Garavaglia E, Tagliani A (1990) Recurrence time distributions: a discussion. In: Proceedings of the 9th ECEE European conference on earthquake engineering, Moscow, September 1990

Jaynes ET (1957) Information theory and statistical mechanics. Phys Rev 106(4):620-630. doi:10.1103/ PhysRev.106.620

Matthews MV, Ellsworth WL, Reasenberg PA (2002) A Brownian model for recurrent earthquakes. Bull Seismol Soc Am 92(6):2233-2250. doi:10.1785/0120010267

Maybeck PS (1979) Stochastic models, estimation, and control, vols I and II. Academic, New York, NJ, USA

Tagliani A (1989) Principle of maximum entropy of probability distributions: definition of applicability field. Probab Eng Mech 4(2):99-104

Tagliani A (1990) On the existence of maximum entropy distributions with four and more assigned moments. Probab Eng Mech 5(4):167-170. doi:10.1016/ 0266-8920(90)90017-E

Vere-Jones D, Ozaki T (1982) Some examples of statistical estimation applied to earthquake data-I. Cyclic Poisson and self exciting models. Ann Inst Stat Math 34(Part B):189-207 